\title{
Implications of the AMS-02 antiproton data for dark matter and cosmic-ray propagation
}

\author{
Hong-Bo Jin \\ National Astronomical Observatories, CAS, Beijing, 100190, P.R. China* \\ Yue-Liang Wu \\ University of Chinese Academy of Sciences \\ Institute of Theoretical Physics, CAS, Beijing, 100190, P.R. China ${ }^{\dagger}$ \\ Yu-Feng Zhou $\ddagger$ \\ Institute of Theoretical Physics, CAS, Beijing, 100190, P.R. China $§$
}

\begin{abstract}
The AMS-02 data on $\bar{p} / p$ flux ratio together with the precision measurements of the proton flux and $\mathrm{B} / \mathrm{C}$ flux ratio provide new insight on the propagation of cosmic rays and can set stringent limits on the dark matter properties. Using propagation models derived from the AMS-02 data alone, one can derive very conservative upper limits on the DM annihilation cross sections. The limits turned out to be compatible with that from the Ferm-LAT gamma-ray data on the dwarf spheroidal satellite galaxies. The flattening of the $\bar{p} / p$ spectrum above $\sim 100 \mathrm{GeV}$ in the current data still leaves some room for TeV scale DM particles. More antiproton data at high kinetic energies are needed to constrain the properties of the DM particles.
\end{abstract}

The 11th International Workshop Dark Side of the Universe 2015

14-18 December 2015

Kyoto, Japan

\footnotetext{
*Email: hbjin@itp.ac.cn

$\dagger$ Email: ylwu@itp.ac.cn

${ }^{\ddagger}$ Speaker.

${ }^{\S}$ Email: yfzhou@itp.ac.cn
} 
Cosmic-ray antiparticles, such as positrons and antiprotons play important roles in the indirect search for dark matter (DM) in the Galactic halo. The Alpha Magnetic Spectrometer (AMS-02) is measuring such cosmic-ray charged particles with unprecedented accuracies. So far the anomalous rise in the positron fraction previous reported by PAMELA ${ }^{1,2}$ and Fermi-LAT ${ }^{3}$ has been confirmed by AMS-02 with higher accuracy and extended to higher energies, ${ }^{4}$ which has triggered extensive theoretical studies on possible explanations including halo DM annihilation or decay (for recent global analyses on AMS-02 data, see e.g. Refs ${ }^{5-15}$ ). Antiprotons are highly expected from DM annihilation in many DM models, which is unlikely to be generated from the nearby pulsars, but can be produced from the collisions inside the supernova remnants.

In 2015, the AMS-02 collaboration released the first preliminary result of the cosmic-ray antiproton to proton flux ratio $\bar{p} / p .{ }^{16}$ The measured kinetic energies of the antiprotons have been extended to $\sim 450 \mathrm{GeV}$. Although the spectrum of $\bar{p} / p$ at high energies above $100 \mathrm{GeV}$ tend to be relatively flat, within uncertainties the AMS-02 data are consistent with the background of secondary antiprotons, which can be used to set stringent upper limits on the dark matter (DM) annihilation cross sections, especially for high mass DM particles. The constraints on the DM properties from antiprotons have been investigated previously before AMS-02 ( see e.g. ${ }^{17-21}$ ). In this talk, we briefly summarise our work on the implications of the new AMS-02 $\bar{p} / p$ data for constraining the annihilation cross sections of the DM particles in various propagation models and DM profiles. The details of the analysis can be found in Ref. ${ }^{22}$

In the diffusion models of cosmic-ray propagation, the Galactic halo within which the diffusion processes occur is parametrized by a cylinder with radius $R_{h}=20-30 \mathrm{kpc}$ and half-height $Z_{h}=$ $3-10 \mathrm{kpc}$. The diffusion equation for the cosmic-ray charged particles reads

$$
\begin{aligned}
\frac{\partial \psi}{\partial t}= & \nabla\left(D_{x x} \nabla \psi-\boldsymbol{V}_{c} \psi\right)+\frac{\partial}{\partial p} p^{2} D_{p p} \frac{\partial}{\partial p} \frac{1}{p^{2}} \psi-\frac{\partial}{\partial p}\left[\dot{p} \psi-\frac{p}{3}\left(\nabla \cdot \boldsymbol{V}_{c}\right) \psi\right] \\
& -\frac{1}{\tau_{f}} \psi-\frac{1}{\tau_{r}} \psi+q(\boldsymbol{r}, p),
\end{aligned}
$$

where $\psi(\boldsymbol{r}, p, t)$ is the number density per unit of total particle momentum. For steady-state diffusion, it is assumed that $\partial \psi / \partial t=0$. The number densities of cosmic-ray particles are assumed to be vanishing at the boundary of the halo. The energy dependent spatial diffusion coefficient $D_{x x}$ is parametrized as $D_{x x}=\beta D_{0}\left(\rho / \rho_{0}\right)^{\delta}$, where $\rho$ is the rigidity of the cosmic-ray particle. The power spectral index $\delta$ can have different values $\delta=\delta_{1(2)}$ for $\rho$ below (above) a reference rigidity $\rho_{0} . D_{0}$ is a normalization constant. The convection term in the diffusion equation is related to the drift of cosmic-ray particles from the Galactic disc due to the Galactic wind. The diffusion in momentum space is described by the reacceleration parameter $D_{p p}$ which is related to the Alfvèn speed $V_{a}$ of disturbances in the hydrodynamical plasma. ${ }^{23}$ The momentum loss rate is denoted by $\dot{p}$, and $\tau_{f}\left(\tau_{r}\right)$ is the time scale for fragmentation (radioactive decay) of the cosmic-ray nuclei.

The spectrum of a primary source term for a cosmic-ray nucleus $A$ is assumed to have a broken power low behaviour $d q_{A}(p) / d p \propto\left(\rho / \rho_{A s}\right)^{\gamma_{A}}$ with $\gamma_{A}=\gamma_{A 1}\left(\gamma_{A 2}\right)$ for the nucleus rigidity $\rho$ below (above) a reference rigidity $\rho_{A s}$. The spatial distribution of the primary sources is assumed to follow that of the pulsars and is taken from Ref. ${ }^{24}$ The background antiproton is assumed to only have the secondary origin, namely, they are created dominantly from inelastic $p p$ - and $p \mathrm{~A}$-collisions with 
the interstellar gas. The corresponding source term reads

$$
q_{\mathrm{sec}}(p)=\beta c n_{i} \sum_{i=\mathrm{H}, \mathrm{He}} \int d p^{\prime} \frac{\sigma_{i}\left(p, p^{\prime}\right)}{d p^{\prime}} n_{p}\left(p^{\prime}\right)
$$

where $n_{i}$ is the number density of the interstellar hydrogen (helium), $n_{p}$ is the number density of primary cosmic-ray proton per total momentum, and $d \sigma_{i}\left(p, p^{\prime}\right) / d p^{\prime}$ is the differential cross section for the antiproton production. In calculating the antiprotons, the inelastic scatterings which produce "tertiary" antiprotons should be taken into account. The primary source from the annihilation of Majorana DM particles has the following form

$$
q_{\mathrm{DM}}(\boldsymbol{r}, p)=\frac{\rho(\boldsymbol{r})^{2}}{2 m_{\chi}^{2}}\langle\sigma v\rangle \sum_{X} \eta_{X} \frac{d N^{(X)}}{d p},
$$

where $\langle\sigma v\rangle$ is the velocity-averaged DM annihilation cross section multiplied by DM relative velocity. $\rho(\boldsymbol{r})$ is the DM energy density distribution function, and $d N^{(X)} / d p$ is the injection energy spectrum of antiprotons from DM annihilating into SM final states through possible intermediate states $X$ with $\eta_{X}$ the corresponding branching fractions. At the top of the atmosphere (TOA) of the Earth, the fluxes of cosmic-rays are affected by solar winds and the helioshperic magnetic field. This effect is taken into account using the force-field approximation which involves the Fisk potential $\phi .{ }^{25}$ We shall take $\phi=550 \mathrm{MV}$ in numerical analysis.

We solve the diffusion equation of Eq. (1) using the numerical code GALPROP v54. ${ }^{26-30} \mathrm{We}$ start with the so-called "conventional" diffusive re-acceleration (DR) model ${ }^{28,30}$ which is commonly adopted by the current experimental collaborations as a benchmark model for the astrophysical backgrounds. It is useful to consider this model as a reference model to understand how the DM properties could be constrained by the AMS-02 data. We then consider three representative propagation models selected from a large sample of models obtained from a global Bayesian MCMC fit to the AMS-02 proton and B/C data using the GALPROP code. ${ }^{31}$ They are selected to represent the typically minimal (MIN), median (MED) and maximal (MAX) antiproton fluxes within $95 \% \mathrm{CL}$, corresponding to the region enveloping $95 \%$ of the MCMC samples with highest likelihoods in a six-dimensional parameter space. Note that the GALPROP based "MIN", "MED" and "MAX" models used in this work are different from and complementary to that given in Ref. ${ }^{32}$ which are based on different theoretical framework.

The predictions for the background of the $\bar{p} / p$ flux ratio in these models are shown in Fig. 1. Note that the "MIN", "MED" and "MAX" models are highly degenerate in the background $\bar{p} / p$ ratio. Compared with these models, the "conventional" model predicts more low energy antiprotons but at high energies above $\sim 500 \mathrm{GeV}$, the predicted antiprotons are much less. In all the four DR propagation models, below $\sim 10 \mathrm{GeV}$ the GALPROP based calculations underpredict the $\bar{p} / p$ flux ratio by $\sim 40 \%$, which is a known issue. The agreement with the low energy $\bar{p}$ data can be improved by introducing breaks in diffusion coefficients, ${ }^{33}$ "fresh" nuclei component ${ }^{34}$ or a DM contribution. ${ }^{17}$ The predictions for low energy $\bar{p} / p$ ratio can also be easily modified by introducing an independent Fisk potential $\phi$ for $\bar{p}$ and an energy-dependent overall normalization factor as discussed in Ref. ${ }^{35}$ We instead use these DR models to derived very conservative upper limits on the annihilation cross sections of light DM particles. Note, however, that in the DR propagation 


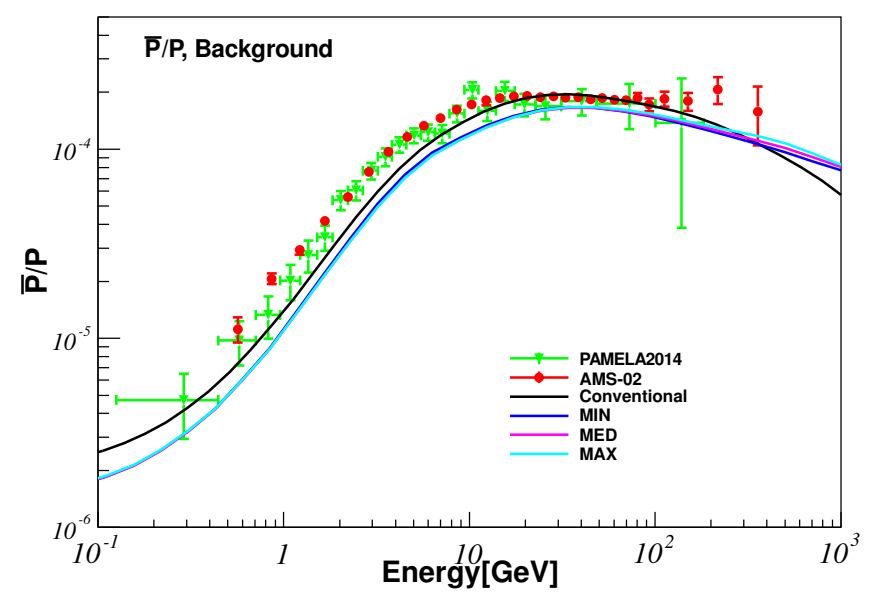

FIG. 1: Predictions for the $\bar{p} / p$ ratio from the four propagation models. The data from AMS- $02^{16}$ and PAMELA $^{36}$ are shown. See text for detailed discription.

models, the background predictions agree with the AMS-02 data well at higher energies $\sim 10-100$ $\mathrm{GeV}$, which can be turned into stringent constraints on the nature of heavy DM particles.

We consider three reference DM annihilation channels $\bar{\chi} \chi \rightarrow \bar{X} X$ where $\bar{X} X=q \bar{q}, b \bar{b}$ and $W^{+} W^{-}$. The energy spectra of these channels are similar at high energies. The main difference is in the average number of total antiprotons $N_{X}$ per DM annihilation of each channel. The injection spectra $d N^{(X)} / d p$ from DM annihilation are calculated using the numerical package PYTHIA v8.175. ${ }^{37}$

We shall first derive upper limits on DM annihilation cross section as a function of DM particle mass, using the frequentist $\chi^{2}$-analyses of the AMS- $02 \bar{p} / p$ data for all of the 30 data points. In Fig. 2, we show the obtained upper limits on the cross sections for DM particle annihilation into $b \bar{b}$ final states from the AMS-02 $\bar{p} / p$ data in the "conventional", "MED", "MIN" and "MAX" propagation models. Four different DM profiles: NFW, ${ }^{38}$ Isothermal, ${ }^{39}$ Einasto $^{40}$ and Moore ${ }^{41,42}$ are considered. As can be seen from the figure, the upper limits as a function of $m_{\chi}$ show some smooth structure for all the final states and DM profiles. The limits tend to be relatively stronger at $m_{\chi} \approx 300 \mathrm{GeV}$, which is related to the fact that the background predictions agree with the data well at the antiproton energy range $\sim 20-100 \mathrm{GeV}$. For a comparison, the upper limits from the FermiLAT 6-year gamma-ray data of the dwarf spheroidal satellite galaxies of the Milky Way ${ }^{43}$ are also shown in Fig. 2. In the "conventional" model, the upper limits from the AMS-02 $\bar{p} / p$ data are found to be compatible with that derived from the Fermi-LAT gamma-ray data for $m_{\chi} \gtrsim 300 \mathrm{GeV}$. This observation is valid for most of the DM profiles. In the "MED" model, the constraints are relatively weaker, which is related to the under prediction of low energy antiprotons in this model and the limits are more conservative. For an estimation of the uncertainties due to the propagation models, from the "MIN" model to the "MAX" model, we find that the variation of the upper limits is within about a factor of five.

For the $W^{+} W^{-}$final states, the resulting limits are shown in Fig. 3. In the "conventional" 

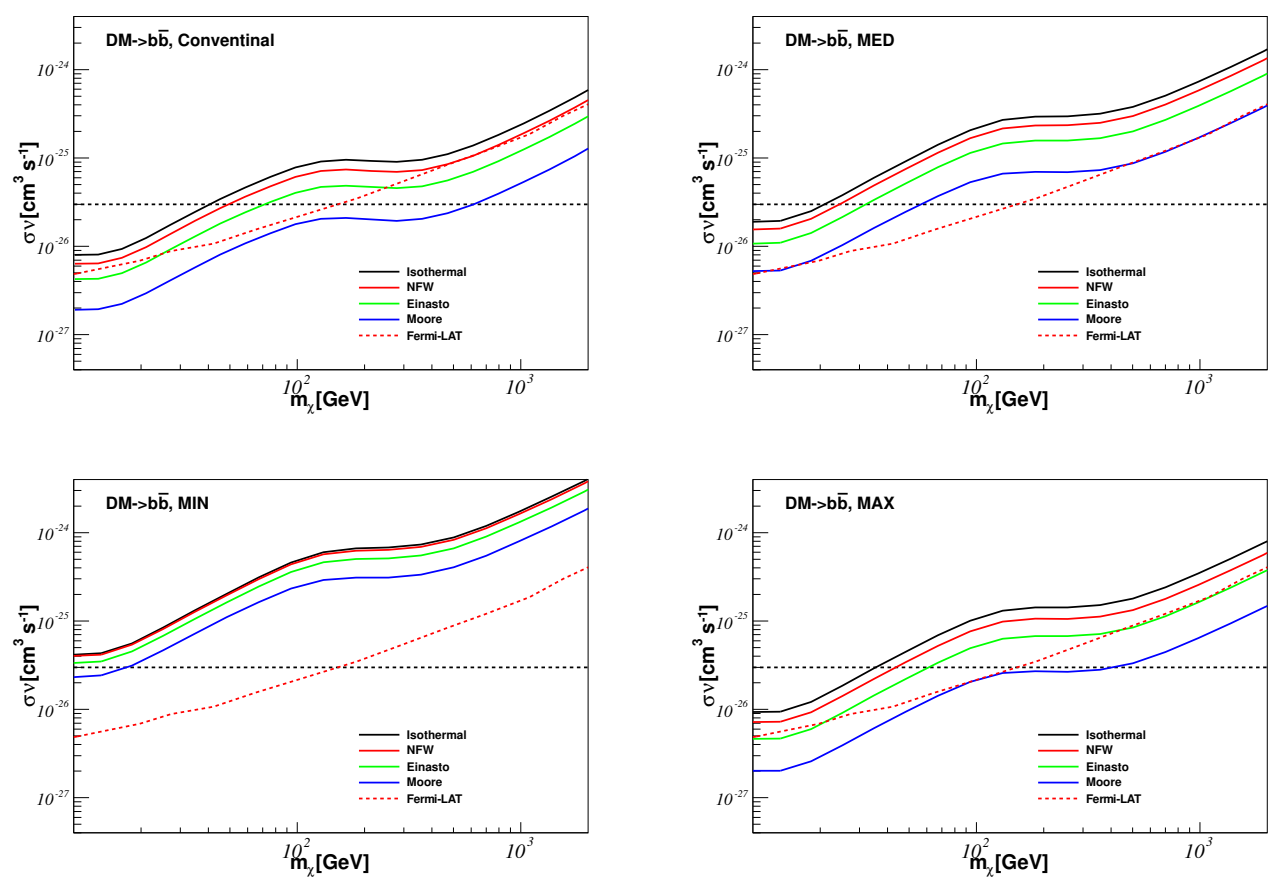

FIG. 2: Upper limits on the cross sections for DM particle annihilation into $b \bar{b}$ final states from the AMS-02 $\bar{p} / p$ data in the "conventional" (upper left), "MED" (upper right), "MIN" (lower left) and "MAX" (lower right) propagation models. Four DM profiles NFW, ${ }^{38}$ Isothermal $^{39}$, Einasto ${ }^{40}$ and Moore ${ }^{41,42}$ are considered. The upper limits from the Fermi-LAT 6-year gamma-ray data of the dwarf spheroidal satellite galaxies of the Milky Way are also shown. ${ }^{43}$ The horizontal line indicates the typical thermal annihilation cross section $\langle\sigma v\rangle=3 \times 10^{-26} \mathrm{~cm}^{3} \mathrm{~s}^{-1}$.

propagation model, the constraints from AMS-02 $\bar{p} / p$ data turn out to be compatible with that from the Fermi-LAT gamma-ray data for all the four DM profiles when the DM particle mass is above $\sim 300 \mathrm{GeV}$. Again we find that the variation of the upper limits from the "MIN" to the "MAX" model is within a factor of five. The result for the $q \bar{q}$ final states is shown in Fig. 4. Compared with the case of $W^{+} W^{-}$and $b \bar{b}$, the constraints on the $q \bar{q}$ final states are the most stringent. For all the three final states, we find that the allowed DM annihilation cross section is below the typical thermal cross section for $m_{\chi} \lesssim 300 \mathrm{GeV}$ in the conventional propagation model with the Einasto DM density profile.

As can be seen clearly in Fig. 1, compared with the AMS-02 data the GALPROP diffusive reacceleration models predict fewer antiprotons at low $(\lesssim 10 \mathrm{GeV})$ and very high $(\gtrsim 100 \mathrm{GeV})$ energies. Without a robust estimation of the theoretical uncertainties, it is too early to claim any excesses in the $\bar{p} / p$ data. We nevertheless consider what would be the implications for DM if such a trend in data is confirmed by future analyses. The low energy data would allow for a nonvanishing DM annihilation cross section. For instance, in the "conventional" propagation model, for $m_{\chi}=10.1,35.0$ and $75.8 \mathrm{GeV}$, the best-fit values are $\langle\sigma v\rangle=3.6 \times 10^{-27}, 1.14 \times 10^{-26}$, and $2.79 \times 10^{-26} \mathrm{~cm}^{3} \mathrm{~s}^{-1}$, respectively, if the DM profile is Einasto, and the DM particles annihilate dominantly into $\bar{b} b$ final states. If both $m_{\chi}$ and $\langle\sigma v\rangle$ are allowed to vary freely, the best-fit DM 

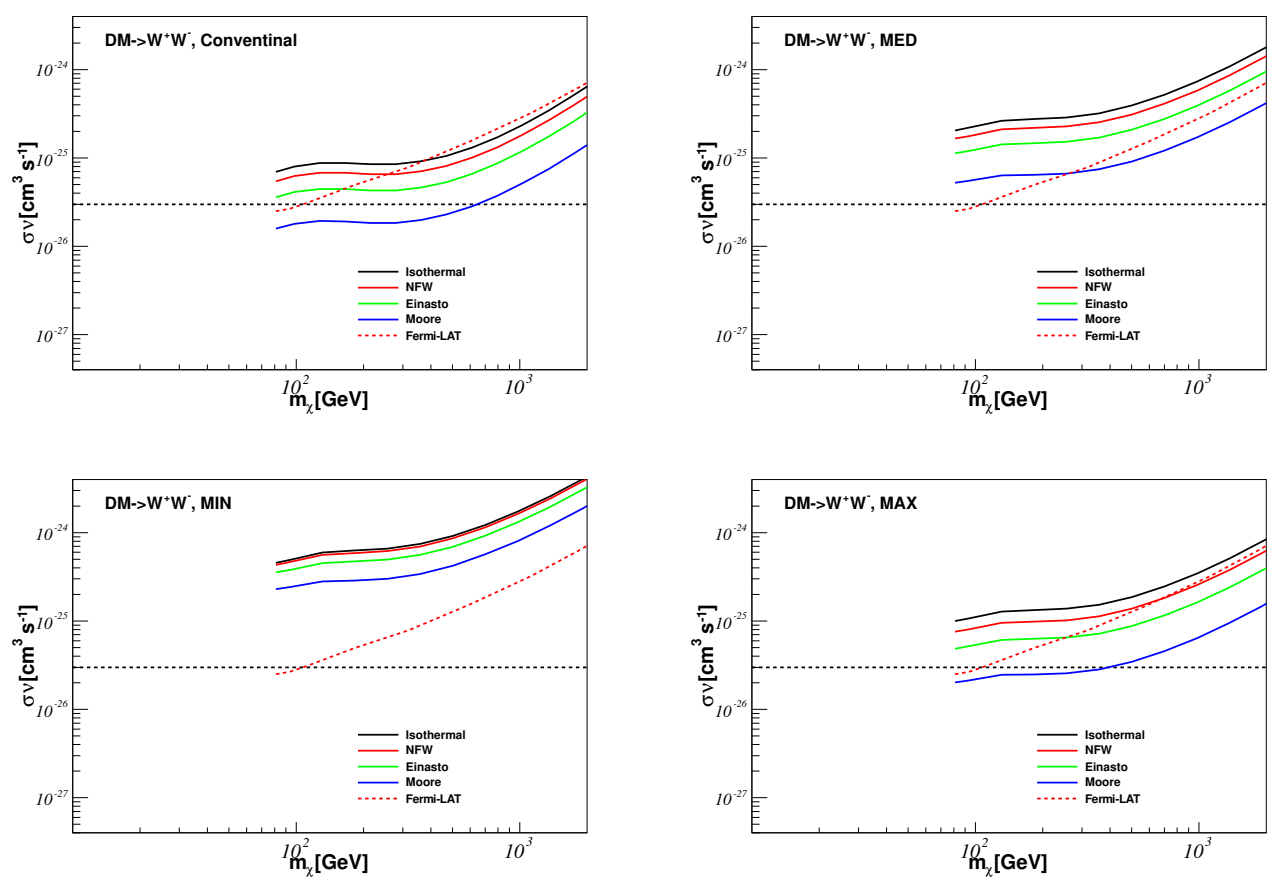

FIG. 3: The same as Fig. 2, but for DM annihilation into $W^{+} W^{-}$final states.

particle masses and annihilation cross sections are $m_{\chi}=58.5(35.0) \mathrm{GeV}$ and $\langle\sigma v\rangle=2.16(0.86) \times$ $10^{-26} \mathrm{~cm}^{3} \mathrm{~s}^{-1}$ for DM annihilating into $\bar{b} b(\bar{q} q)$ final states. In Fig. 5, we show the calculated spectra of $\bar{p} / p$ flux ratio from the best-fit DM particle masses and cross sections. The figure shows that the low energy $\bar{p} / p$ data are well reproduced by including such a DM contribution, except for the data point with kinetic energy below $1 \mathrm{GeV}$. Note that the predictions for low energy $\bar{p} / p$ suffer from considerable astrophysical uncertainties. Such an analysis is only for a reference.

As shown in Fig. 1, the spectrum of the AMS- $02 \bar{p} / p$ ratio tends to be flat toward high energies above $\sim 100 \mathrm{GeV}$. This trend, if confirmed by the future AMS-02 data, is not expected from the secondary production of antiprotons, and raises the interesting question whether this would leave some room for a heavy DM contribution, similar to the case of the AMS-02 positron fraction. ${ }^{4,5,9-11}$ To explore this possibility, we perform fits using the $\bar{p} / p$ ratio data above $20 \mathrm{GeV}$ (including 15 data points in total) in order to avoid the theoretical uncertainties in the low energy region. The obtained $\chi^{2}$ curve, i.e., $\chi_{\min }^{2}$ as a function of $m_{\chi}$ for the $b \bar{b}, q \bar{q}$ and $W^{+} W^{-}$final states in the "conventional" propagation model with Einasto DM profile are shown in Fig. 6. For the three final states the values of $\chi_{\min }^{2}$ decrease almost monotonically from $\sim 21$ to $\sim 5$ with an increasing DM particles mass from $100 \mathrm{GeV}$ to $10 \mathrm{TeV}$, but the $\chi^{2}$-curves gradually flatten out toward high DM masses. From the $\chi^{2}$-curves, one can see that the DM particles mass is restricted to be above $\sim 2 \mathrm{TeV}$ at $2 \sigma$. For an illustration purpose, we show in Fig. 6 the predictions for the $\bar{p} / p$ ratio in the "conventional" background model with a DM contribution. The DM particles masses and annihilation cross sections chosen to be $m_{\chi}=6.5 \mathrm{TeV},\langle\sigma v\rangle=1.9 \times 10^{-24} \mathrm{~cm}^{3} \mathrm{~s}^{-1}$ for $W^{+} W^{-}$, $m_{\chi}=10.9 \mathrm{TeV},\langle\sigma v\rangle=3.4 \times 10^{-24} \mathrm{~cm}^{3} \mathrm{~s}^{-1}$ for $b \bar{b}$ channel, and $m_{\chi}=10.9 \mathrm{TeV}$ and $\langle\sigma v\rangle=$ 

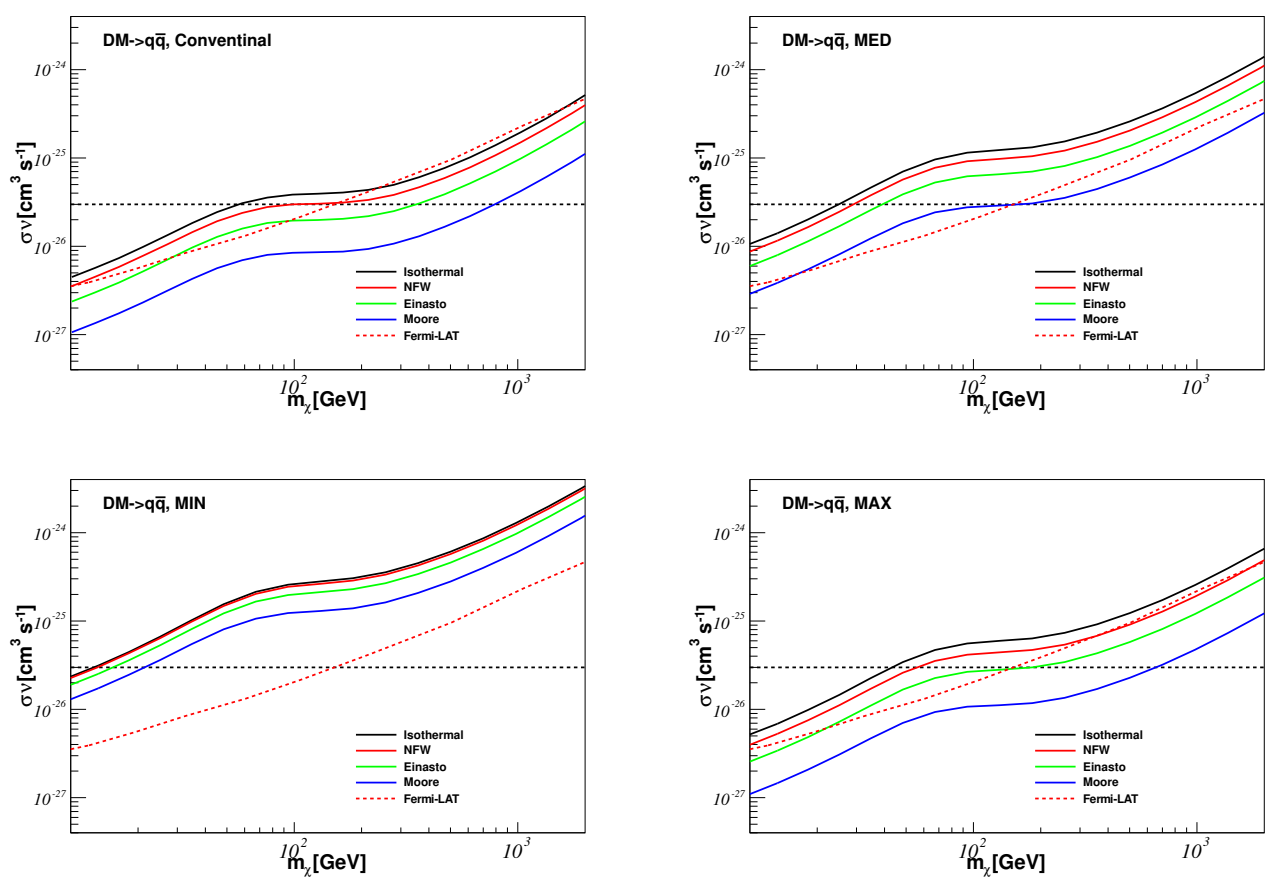

FIG. 4: The same as Fig. 2, but for DM annihilation into $q \bar{q}$ final states.

$3.3 \times 10^{-24} \mathrm{~cm}^{3} \mathrm{~s}^{-1}$ for $q \bar{q}$ channel. Note that these values are not from the best-fit values. We conclude that introducing a DM contribution can improve the agreement with the AMS-02 $\bar{p} / p$ data with kinetic energy above $100 \mathrm{GeV}$, but the statistics is not high enough to determine the DM properties such as its mass and interaction strength. As can be see in Fig. 1, the possible "excess" is located at the kinetic energy range $100-450 \mathrm{GeV}$ where the secondary backgrounds from the four propagation models are similar. However, beyond $\sim 450 \mathrm{GeV}$, the $\bar{p} / p$ from the "conventional" model drops quicker than that in the other propagation models. The future high energy antiproton data will be very important not only in probing DM but also in constraining the background models.

In summary, we have derived the upper limits using the GALPROP code and shown that in the "conventional " propagation model with Einasto DM profile, the constraints can be more stringent than that derived from the Ferm-LAT gamma-ray data on the dwarf spheroidal satellite galaxies. Making use of the typical minimal, median and maximal models obtained from a previous global fit, we have shown that the uncertainties on the upper limits is around a factor of five. The future more precise AMS-02 data can help to reduce the uncertainties in the derived upper limits. Similar discussions on the DM matter contributions can be found in Refs. ${ }^{44,45}$ So far the analysis is limited to the case of DM direct annihilation into the SM final states. In many well-motivated models, the DM particles interact through mediators, which has reach phenomenological implications, such as modifying DM annihilation cross sections, ${ }^{10,46-48}$ changing the interpretation of the DM direct detection, ${ }^{49}$ diphoton production at colliders ${ }^{50,51}$ and solar capture of DM particles, ${ }^{52,53}$ etc. The presence of mediators may also change the energy spectrum of antiprotons, which will be investigated in a forthcoming publication. 

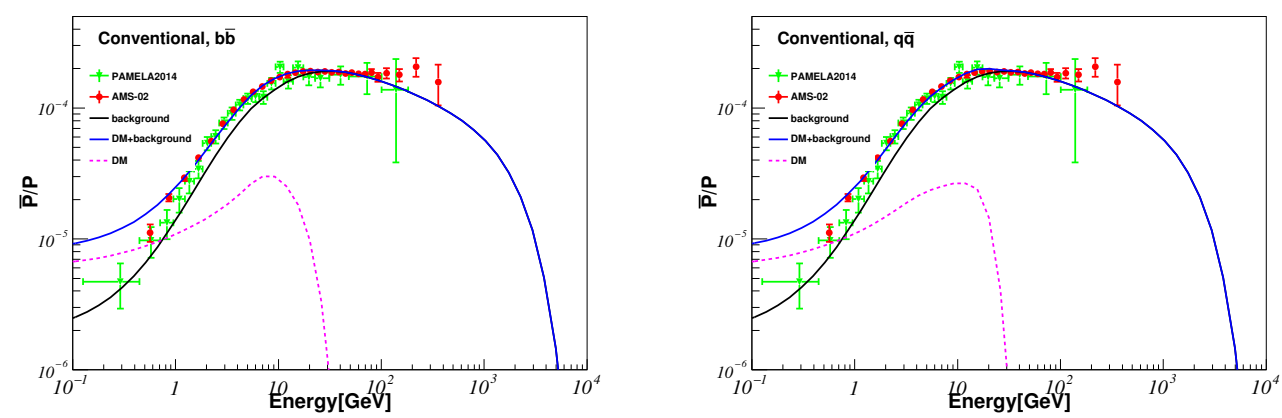

FIG. 5: Left) Spectrum of $\bar{p} / p$ flux ratio from DM annihilating into $\bar{b} b$ final states with $m_{\chi}=58.5 \mathrm{GeV}$ and $\langle\sigma v\rangle=2.16 \times 10^{-26} \mathrm{~cm}^{3} \mathrm{~s}^{-1}$ obtained from a fit to the whole AMS- $02 \bar{p} / p$ data. ${ }^{16}$ The "conventional" background model and the Einasto DM profile are assumed. Right) The same as left, but for the fit with $\bar{q} q$ final state with the best-fit values $m_{\chi}=35 \mathrm{GeV}$ and $\langle\sigma v\rangle=0.86 \times 10^{-26} \mathrm{~cm}^{3} \mathrm{~s}^{-1}$.

This work is supported in part by the National Basic Research Program of China (973 Program) under Grants No. 2010CB833000; the National Nature Science Foundation of China (NSFC) under Grants No. 10905084, No. 11335012 and No. 11475237;

\section{References}

[1] PAMELA Collaboration, O. Adriani et al., An anomalous positron abundance in cosmic rays with energies 1.5-100 GeV, Nature 458 (2009) 607-609, [arXiv : 0810 . 4995].

[2] O. Adriani, G. Barbarino, G. Bazilevskaya, R. Bellotti, M. Boezio, et al., A statistical procedure for the identification of positrons in the PAMELA experiment, Astropart.Phys. 34 (2010) 1-11, [arXiv:1001.3522].

[3] Fermi-LAT Collaboration, M. Ackermann et al., Measurement of separate cosmic-ray electron and positron spectra with the Fermi Large Area Telescope, Phys.Rev.Lett. 108 (2012) 011103, [arXiv:1109.0521].

[4] AMS Collaboration, L. Accardo et al., High Statistics Measurement of the Positron Fraction in Primary Cosmic Rays of $0.51 \mathrm{C} 500 \mathrm{GeV}$ with the Alpha Magnetic Spectrometer on the International Space Station, Phys.Rev.Lett. 113 (2014) 121101.

[5] J. Kopp, Constraints on dark matter annihilation from AMS-02 results, Phys.Rev. D88 (2013) 076013, [arXiv:1304.1184].

[6] A. De Simone, A. Riotto, and W. Xue, Interpretation of AMS-02 Results: Correlations among Dark Matter Signals, JCAP 1305 (2013) 003, [arXiv: 1304 .1336].

[7] I. Cholis and D. Hooper, Dark Matter and Pulsar Origins of the Rising Cosmic Ray Positron Fraction in Light of New Data From AMS, Phys.Rev. D88 (2013) 023013, [arXiv: 1304 . 1840 ].

[8] L. Feng, R.-Z. Yang, H.-N. He, T.-K. Dong, Y.-Z. Fan, et al., AMS-02 positron excess: new bounds on dark matter models and hint for primary electron spectrum hardening, Phys.Lett. B728 (2014) 250-255, [arXiv:1303.0530].

[9] H.-B. Jin, Y.-L. Wu, and Y.-F. Zhou, Implications of the first AMS-02 measurement for dark matter annihilation and decay, JCAP 1311 (2013) 026, [arXiv: 1304 . 1997]. 

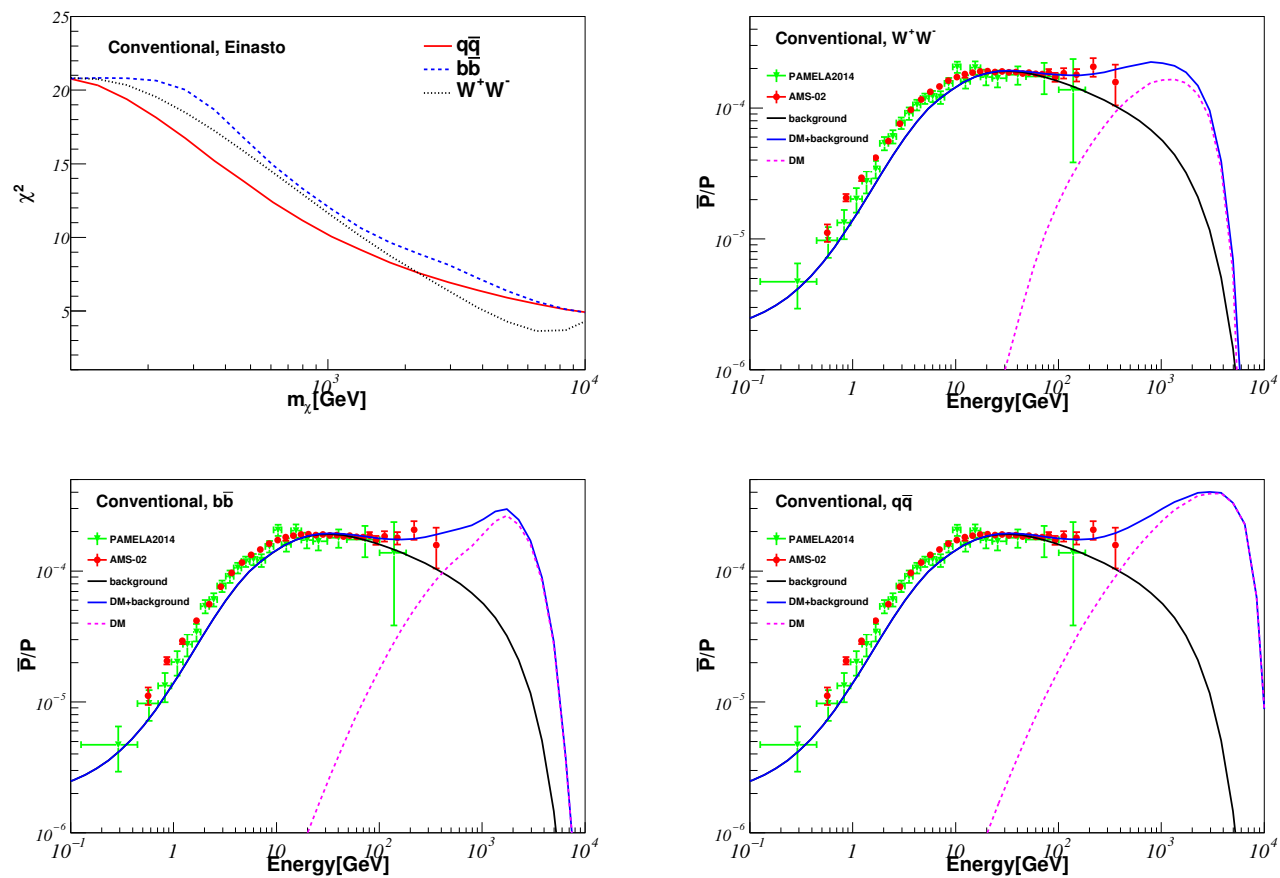

FIG. 6: (Upper left) values of $\chi_{\min }^{2}$ as a function of DM particle mass $m_{\chi}$ from a fit to the AMS-02 $\bar{p} / p$ data ( with kinetic energy above $20 \mathrm{GeV}$ ) in the "conventional" propagation model ${ }^{28,30}$ with the DM profile fixed to Einasto. ${ }^{40}$ Three annihilation channels $b \bar{b}, q \bar{q}$ and $W^{+} W^{-}$are considered. (Upper right) predicted $\bar{p} / p$ ratio in the case of background ("conventional" model) plus a DM contribution with $m_{\chi}=6.5 \mathrm{TeV}$, $\langle\sigma v\rangle=1.9 \times 10^{-24} \mathrm{~cm}^{3} \mathrm{~s}^{-1}$, and annihilation final states $W^{+} W^{-}$. The flux ratio of antiproton from DM to the proton from the background $\bar{p}_{\mathrm{DM}} / p_{\mathrm{BG}}$ is shown as the dashed line. The data from AMS- $02^{16}$ and PAMELA $^{36}$ are also shown. (Lower left) the same as the upper right, but for the $b \bar{b}$ channel with $m_{\chi}=$ $10.9 \mathrm{TeV}$ and $\langle\sigma v\rangle=3.4 \times 10^{-24} \mathrm{~cm}^{3} \mathrm{~s}^{-1}$. (Lower right) the same as the upper right, but for the $q \bar{q}$ channel with $m_{\chi}=10.9 \mathrm{TeV}$ and $\langle\sigma v\rangle=3.3 \times 10^{-24} \mathrm{~cm}^{3} \mathrm{~s}^{-1}$.

[10] Z.-P. Liu, Y.-L. Wu, and Y.-F. Zhou, Sommerfeld enhancements with vector, scalar and pseudoscalar force-carriers, Phys.Rev. D88 (2013) 096008, [arXiv:1305. 5438].

[11] L. Bergstrom, T. Bringmann, I. Cholis, D. Hooper, and C. Weniger, New limits on dark matter annihilation from AMS cosmic ray positron data, Phys.Rev.Lett. 111 (2013) 171101, [arXiv:1306.3983].

[12] A. Ibarra, A. S. Lamperstorfer, and J. Silk, Dark matter annihilations and decays after the AMS-02 positron measurements, Phys.Rev. D89 (2014), no. 6 063539, [arXiv:1309.2570].

[13] M. Di Mauro, F. Donato, N. Fornengo, R. Lineros, and A. Vittino, Interpretation of AMS-02 electrons and positrons data, JCAP 1404 (2014) 006, [arXiv: 1402 .0321].

[14] S.-J. Lin, Q. Yuan, and X.-J. Bi, Quantitative study of the AMS-02 electron/positron spectra: Implications for pulsars and dark matter properties, Phys.Rev. D91 (2015), no. 6 063508, [arXiv:1409.6248].

[15] M. Ibe, S. Matsumoto, S. Shirai, and T. T. Yanagida, Mass of Decaying Wino from AMS-02 2014, Phys.Lett. B741 (2015) 134-137, [arXiv: 1409.6920 ]. 
[16] S.Ting, talk at AMS-02 days at CERN, April 15-17, CERN, Geneva, https://indico.cern.ch/event/381134/timetable/\#20150415.

[17] D. Hooper, T. Linden, and P. Mertsch, What Does The PAMELA Antiproton Spectrum Tell Us About Dark Matter?, JCAP 1503 (2015), no. 03 021, [arXiv: 1410.1527 ].

[18] R. Kappl and M. W. Winkler, The Cosmic Ray Antiproton Background for AMS-02, JCAP 1409 (2014) 051, [arXiv:1408.0299].

[19] N. Fornengo, L. Maccione, and A. Vittino, Constraints on particle dark matter from cosmic-ray antiprotons, JCAP 1404 (2014) 003, [arXiv: 1312 . 3579].

[20] M. Cirelli and G. Giesen, Antiprotons from Dark Matter: Current constraints and future sensitivities, JCAP 1304 (2013) 015, [arXiv:1301. 7079].

[21] H.-B. Jin, S. Miao, and Y.-F. Zhou, Implications of the latest XENON100 and cosmic ray antiproton data for isospin violating dark matter, Phys.Rev. D87 (2013), no. 1 016012, [arXiv: 1207.4408 ].

[22] H.-B. Jin, Y.-L. Wu, and Y.-F. Zhou, Upper limits on DM annihilation cross sections from the first AMS-02 antiproton data, arXiv:1504.04604.

[23] V. Ginzburg, V. Dogiel, V. Berezinsky, S. Bulanov, and V. Ptuskin, Astrophysics of cosmic rays, .

[24] A. Strong and I. Moskalenko, Propagation of cosmic-ray nucleons in the galaxy, Astrophys.J. 509 (1998) 212-228, [astro-ph/9807150].

[25] L. Gleeson and W. Axford, Solar Modulation of Galactic Cosmic Rays, Astrophys.J. 154 (1968) 1011.

[26] A. Strong and I. Moskalenko, Propagation of cosmic-ray nucleons in the galaxy, Astrophys.J. 509 (1998) 212-228, [astro-ph/9807150].

[27] I. V. Moskalenko, A. W. Strong, J. F. Ormes, and M. S. Potgieter, Secondary anti-protons and propagation of cosmic rays in the galaxy and heliosphere, Astrophys.J. 565 (2002) 280-296, [astro-ph/0106567].

[28] A. Strong and I. Moskalenko, Models for galactic cosmic ray propagation, Adv.Space Res. 27 (2001) 717-726, [astro-ph/0101068].

[29] I. V. Moskalenko, A. Strong, S. Mashnik, and J. Ormes, Challenging cosmic ray propagation with antiprotons. Evidence for a fresh nuclei component?, Astrophys.J. 586 (2003) 1050-1066, [astro-ph/0210480].

[30] V. Ptuskin, I. V. Moskalenko, F. Jones, A. Strong, and V. Zirakashvili, Dissipation of magnetohydrodynamic waves on energetic particles: impact on interstellar turbulence and cosmic ray transport, Astrophys.J. 642 (2006) 902-916, [astro-ph/ 0510335$].$

[31] H.-B. Jin, Y.-L. Wu, and Y.-F. Zhou, Cosmic ray propagation and dark matter in light of the latest AMS-02 data, arXiv:1410.0171.

[32] F. Donato, N. Fornengo, D. Maurin, and P. Salati, Antiprotons in cosmic rays from neutralino annihilation, Phys.Rev. D69 (2004) 063501, [astro-ph/ 0306207 ].

[33] I. V. Moskalenko, A. W. Strong, J. F. Ormes, and M. S. Potgieter, Secondary anti-protons and propagation of cosmic rays in the galaxy and heliosphere, Astrophys.J. 565 (2002) 280-296, [astro-ph/0106567].

[34] I. V. Moskalenko, A. Strong, S. Mashnik, and J. Ormes, Challenging cosmic ray propagation with antiprotons. Evidence for a fresh nuclei component?, Astrophys.J. 586 (2003) 1050-1066, [astro-ph/0210480]. 
[35] G. Giesen, M. Boudaud, Y. Genolini, V. Poulin, M. Cirelli, et al., AMS-02 antiprotons, at last! Secondary astrophysical component and immediate implications for Dark Matter, arXiv:1504.04276.

[36] O. Adriani, G. Barbarino, G. Bazilevskaya, R. Bellotti, M. Boezio, et al., The PAMELA Mission: Heralding a new era in precision cosmic ray physics, Phys.Rept. 544 (2014) 323-370.

[37] T. Sjostrand, S. Mrenna, and P. Z. Skands, A Brief Introduction to PYTHIA 8.1, Comput.Phys.Commun. 178 (2008) 852-867, [arXiv: 0710.3820 ].

[38] J. F. Navarro, C. S. Frenk, and S. D. White, A Universal density profile from hierarchical clustering, Astrophys.J. 490 (1997) 493-508, [astro-ph/9611107].

[39] L. Bergstrom, P. Ullio, and J. H. Buckley, Observability of gamma-rays from dark matter neutralino annihilations in the Milky Way halo, Astropart.Phys. 9 (1998) 137-162, [astro-ph/9712318].

[40] J. Einasto, Dark Matter, arXiv:0901.0632.

[41] B. Moore, S. Ghigna, F. Governato, G. Lake, T. R. Quinn, et al., Dark matter substructure within galactic halos, Astrophys.J. 524 (1999) L19-L22, [astro-ph/9907411].

[42] J. Diemand, B. Moore, and J. Stadel, Convergence and scatter of cluster density profiles, Mon.Not.Roy.Astron.Soc. 353 (2004) 624, [a stro-ph/ 0402267 ].

[43] Fermi-LAT Collaboration, M. Ackermann et al., Searching for Dark Matter Annihilation from Milky Way Dwarf Spheroidal Galaxies with Six Years of Fermi-LAT Data, arXiv: 1503.02641.

[44] M. Ibe, S. Matsumoto, S. Shirai, and T. T. Yanagida, Wino Dark Matter in light of the AMS-02 2015 Data, Phys. Rev. D91 (2015), no. 11 111701, [arXiv: 1504.05554 ].

[45] C.-H. Chen, C.-W. Chiang, and T. Nomura, Dark matter for excess of AMS-02 positrons and antiprotons, Phys. Lett. B747 (2015) 495-499, [arXiv: 1504.07848 ].

[46] J. Chen and Y.-F. Zhou, The 130 GeV gamma-ray line and Sommerfeld enhancements, JCAP 1304 (2013) 017, [arXiv:1301.5778].

[47] T. Li and Y.-F. Zhou, Strongly first order phase transition in the singlet fermionic dark matter model after LUX, JHEP 07 (2014) 006, [arXiv: 1402 . 3087].

[48] B. Li and Y.-F. Zhou, Direct detection of dark matter with resonant annihilation, Commun. Theor. Phys. 64 (2015), no. 1 119-126, [arXiv: 1503.08281$].$

[49] T. Li, S. Miao, and Y.-F. Zhou, Light mediators in dark matter direct detections, JCAP 1503 (2015), no. 03 032, [arXiv:1412.6220].

[50] X.-J. Huang, W.-H. Zhang, and Y.-F. Zhou, Connecting the LHC diphoton excess to the Galatic center gamma-ray excess, Phys. Rev. D94 (2016), no. 3 035019, [arXiv: 1605.09018 ].

[51] X.-J. Huang, W.-H. Zhang, and Y.-F. Zhou, A 750 GeV dark matter messenger at the Galactic Center, Phys. Rev. D93 (2016) 115006, [arXiv:1512.08992].

[52] Z.-L. Liang, Y.-L. Wu, Z.-Q. Yang, and Y.-F. Zhou, On the evaporation of solar dark matter: spin-independent effective operators, JCAP 1609 (2016), no. 09 018, [arXiv: 1606.02157 ].

[53] J. Chen, Z.-L. Liang, Y.-L. Wu, and Y.-F. Zhou, Long-range self-interacting dark matter in the Sun, JCAP 1512 (2015), no. 12 021, [arXiv: 1505.04031 ]. 